\title{
Negative Improvements, Relative Validity and Elusive Goodness
}

\author{
Matej Batič, Gabriela Hoff, Chan Hyeong Kim, Sung Hun Kim, Maria Grazia Pia, Paolo Saracco and Georg \\ Weidenspointner
}

\begin{abstract}
Various issues related to the complexity of appraising the capabilities of physics models implemented in Monte Carlo simulation codes and the evolution of the functional quality the associated software are considered, such as the dependence on the experimental environment where the software operates and its sensitivity to detector characteristics. The concept of software validity as relative to the environment is illustrated by means of a real-life experimental test case. Methods and techniques to mitigate the risk of deteriorating the quality of the software are critically discussed: they concern various disciplines of the software development process. Quantitative validation of physics models is advocated as a method to appraise their capabilities objectively and to monitor the evolution of their associated software behavior.
\end{abstract}

\section{INTRODUCTION}

$\mathbf{T}$ HE assessment of the quality of physics simulation is an important issue in experimental practice. Experimentalists face the dilemma of selecting, among various Monte Carlo codes and the collection of physics modeling options implemented in them, the most appropriate for their experimental scenario. Often such a choice, which may have a critical impact on an experimental project, cannot be supported by objective arguments, due to the limited availability in the literature of documented, quantitative assessments of the accuracy of the physics models implemented in Monte Carlo codes.

Some of the particle transport systems currently in use are the result of decades of evolution, during which not only physics modeling approaches, but also the software design, the implementation and computational platforms where the software operates have changed with respect to the original configuration in which a Monte Carlo simulation system was first deployed. As a result of this evolution, the original physical behaviour of the software may have been altered: although

Manuscript received 15 November 2013.

This work has been partly funded by CNPq BEX6460/10-0 grant, Brazil.

T. Basaglia is with CERN, CH-1211, Geneva, Switzerland (e-mail: Tullio.Basaglia@cern.ch).

M. Batič was with INFN Sezione di Genova, Genova, Italy (e-mail: Batic.Matej@gmail.com); he is now with Sinergise, 1000 Ljubljana, Slovenia.

C. H. Kim and S. H. Kim are with the Department of Nuclear Engineering, Hanyang University, Seoul 133-791, Korea (e-mail: mchan@hanyang.ac.kr, chkim@hanyang.ac.kr, hsungman@naver.com).

G. Hoff is with Pontificia Universidade Catolica do Rio Grande do Sul, Brazil (e-mail:ghoff.gesic@gmail.com).

M. G. Pia and P. Saracco are with INFN Sezione di Genova, Via Dodecaneso 33, I-16146 Genova, Italy (phone: +39 010 3536328, fax: +39 010 313358, e-mail: MariaGrazia.Pia@ge.infn.it, Paolo.Saracco@ge.infn.it).

G. Weidenspointner is with the Max-Planck-Institut für extraterrestrische Physik, 85740 Garching, Germany (e-mail: Georg.Weidenspointner@hll.mpg.de).

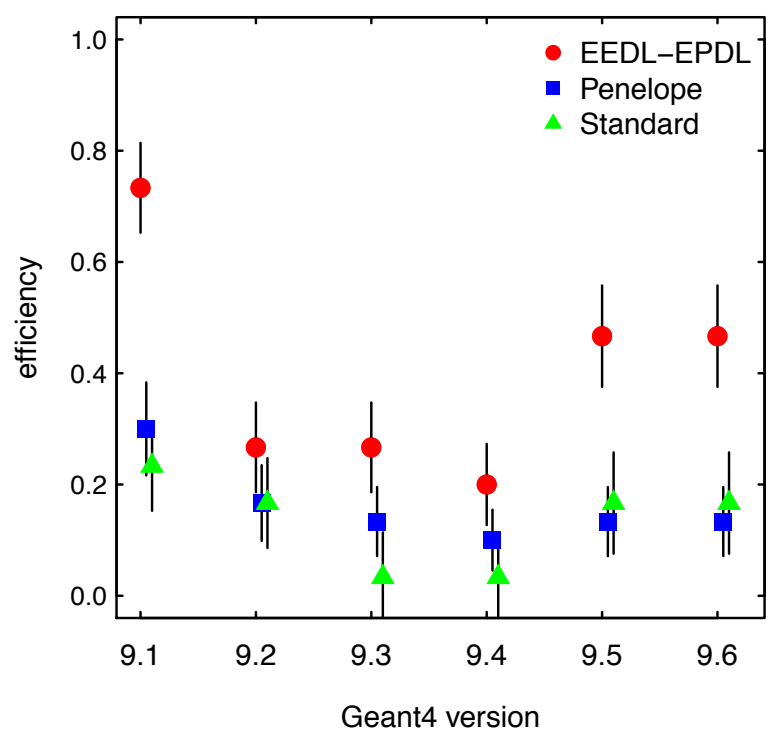

Fig. 1. Fraction of test cases in which Geant4-based simulations of the energy deposited by electrons as a function of penetration depth in matter is found compatible with experimental data at 0.01 significance level, versus Geant 4 version. The experimental data are from [4]. Three Geant4 electromagnetic models (EEDL-EPDL, Penelope and Standard) were compared with experimental measurements.

in conventional wisdom more recent versions of software products are assumed to be of superior quality than older ones, it may occur that in some areas the functional quality of the software actually deteriorates in the course of evolution. Also in this context the lack of quantitative documentation of physics modeling accuracy hinders the discernment between genuine improvements of the code and possible slips in the quality of the software.

Some concrete cases are discussed here, with the intent of providing a constructive contribution to identify possible causes of the deterioration of software functionality, and means to address them effectively. Contributions of various disciplines in the software development to the overall software quality are pondered: they concern not only testing and quality assurance, but also domain decomposition, software design and change management, whose contribution to the functional quality of the software is often neglected. This reflection is especially relevant to experimental environments, where widely used tools and the software of experiments are expected to 


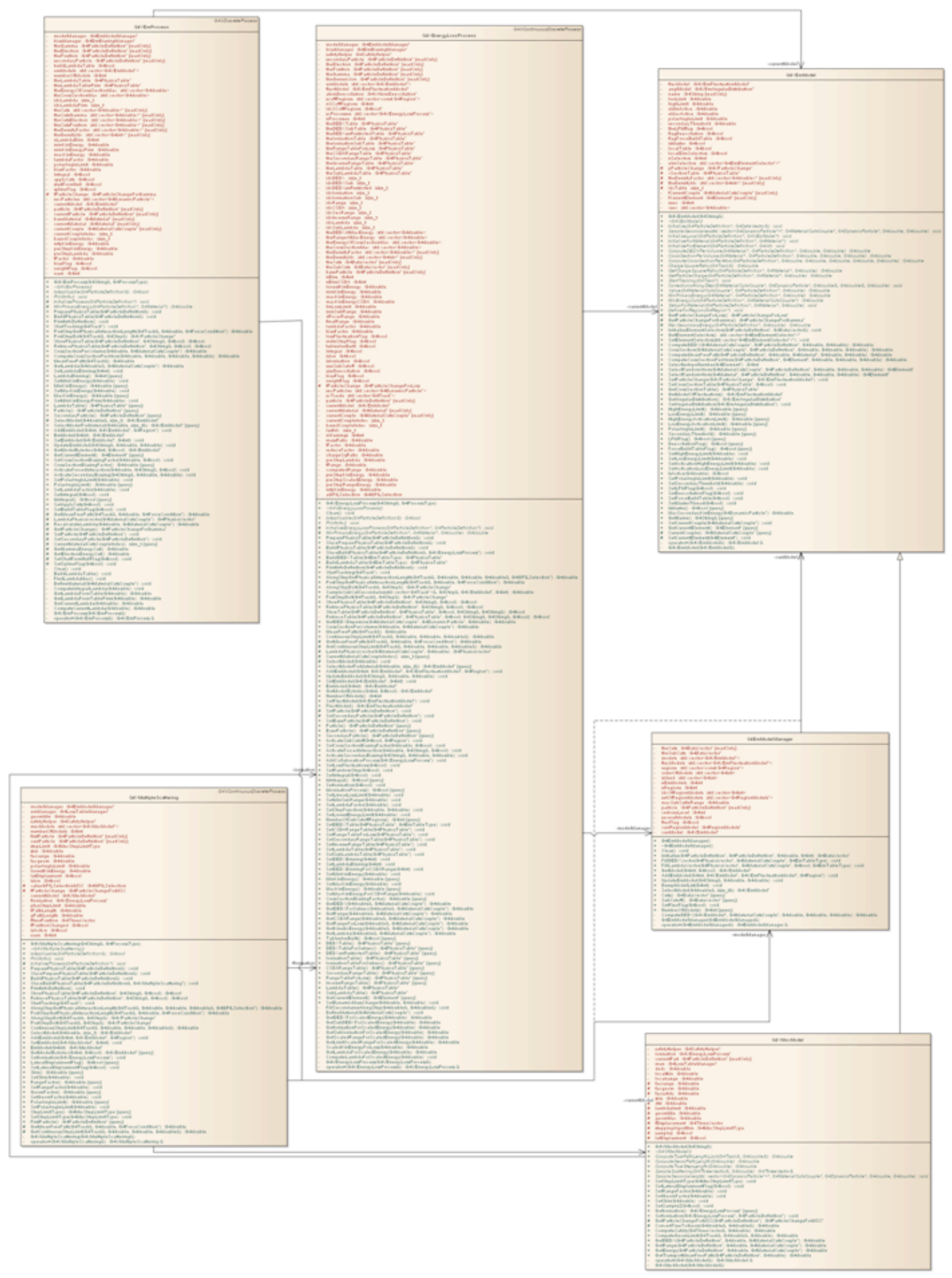

Fig. 2. UML (Unified Modeling Language) class diagram illustrating some features of the design of Geant4 electromagentic processes and models. Class attributes appear in red, class operations in green. The font size in the UML diagram had to be reduced to fit into a page, thus making the details of operations and attributes unreadable; nevertheless, the focus in this diagram should be on the large number of operations and attributes in abstract base classes, rether than on the details of their signatures. 


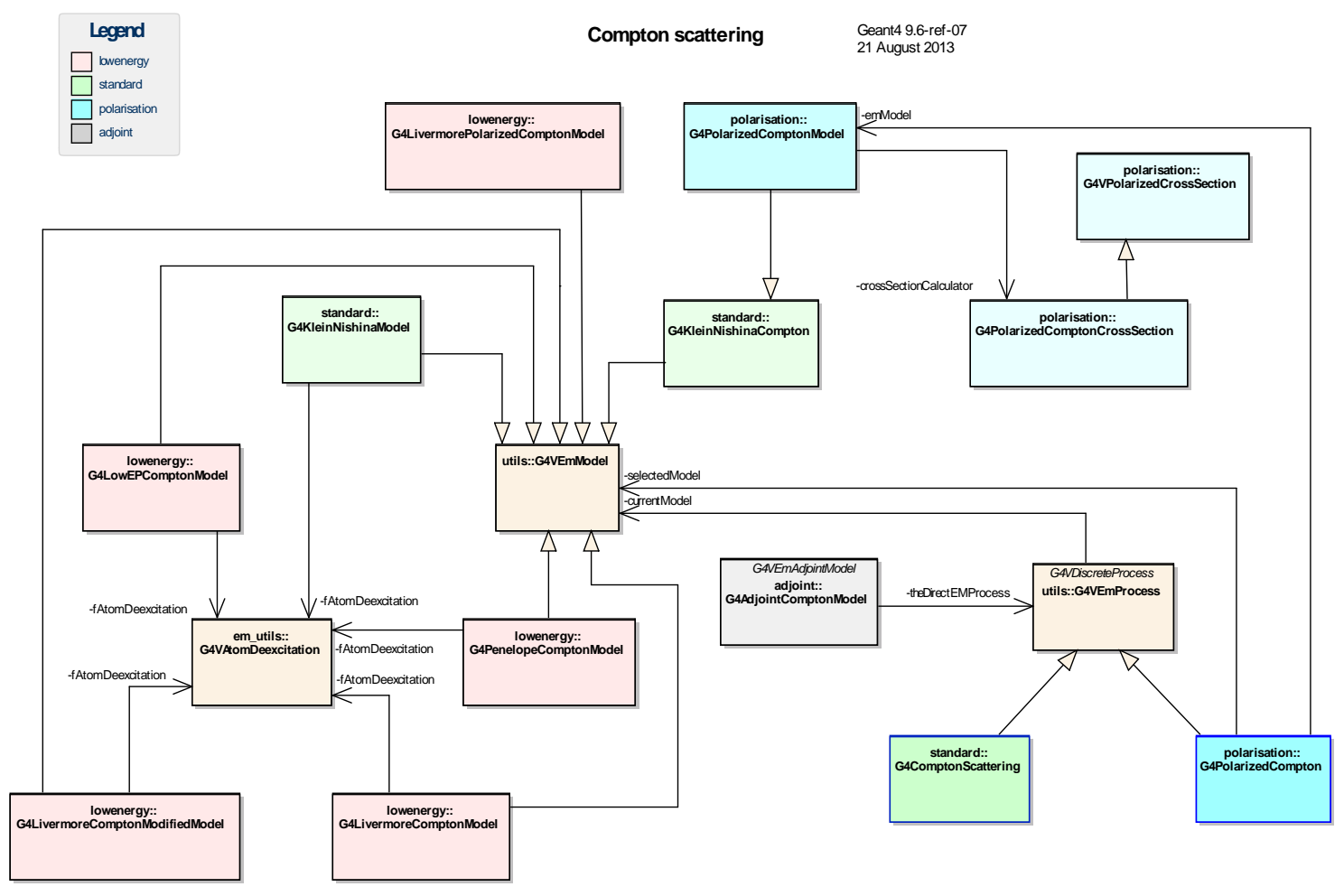

Fig. 3. UML (Unified Modeling Language) class diagram illustrating the software design for the simulation of Compton scattering in Geant 4.

stand long life-cycles, and are necessarily subject to evolution.

\section{Negative improvements}

An extensively documented, quantitative study of software evolution resulting in deterioration of physical accuracy over the years is reported in [1]. The analysis concerns the simulation of the energy deposited by electrons in various experimental configurations resulting from Geant4 [2], [3] versions released between 2007 and 2013. A representative set of results is shown in Fig. 1. which reports the fraction of test cases where longitudinal energy deposition patterns were found statistically compatible with high precision experimental measurements [4]. The evolution of the functional quality of the software is objectively quantified by means of a rigorous statistical analysis, which combines goodness-of-fit tests and methods of categorical data testing to validate the simulation against experimental data. Significantly lower compatibility with experiment is observed with the later Geant 4 versions subject to evaluation; the significance level of the test is 0.01 .

Although [1] stresses that the observed negative improvement concerns a specific observable and a limited software domain, one may wonder what causes could have concurred to achieve the observed effect. The observation of negative improvements in publicly released code hints to some deficiencies in the test and quality assurance process, which could have detected suspicious behaviour in the course of the development process prior to the deployment of the code. While the role of the testing and quality assurance processes may appear obvious in the assessment of the physical accuracy of the code, one should consider also the role of other disciplines, which may facilitate - or hinder - the test process. For instance, a software design characterized by classes charged with multiple responsibilities, extensive inherited behavior and numerous dependencies (an example of which is illustrated in Fig. 2), contributes an unnecessary burden to the test process, as it impedes agile unit tests as a means to assess and monitor the behaviour of physics components. Code duplication, as observed, for instance, in some areas of Geant4 electromagnetic domain, is prone to generate problems of maintenance and evolution of the software [5]: an example in Geant4 electromagnetic physics domain is highlighted in Fig. 3, where a proliferation of Compton scattering models involves duplication of code and physics functionality over different classes.

\section{RELATIVE VALIDITY}

An important issue in software validation, which is often neglected, is specified in the IEEE Standard for Software Verification and Validation [7], which conforms to ISO/IEC 15288 and ISO/IEC 12207 Standards: the validation process should provide evidence that the software satisfies "intended use and user needs". This clause means that the validity of the physics models embedded in a simulation system may be relative to the experimental environment where they are exercised. 


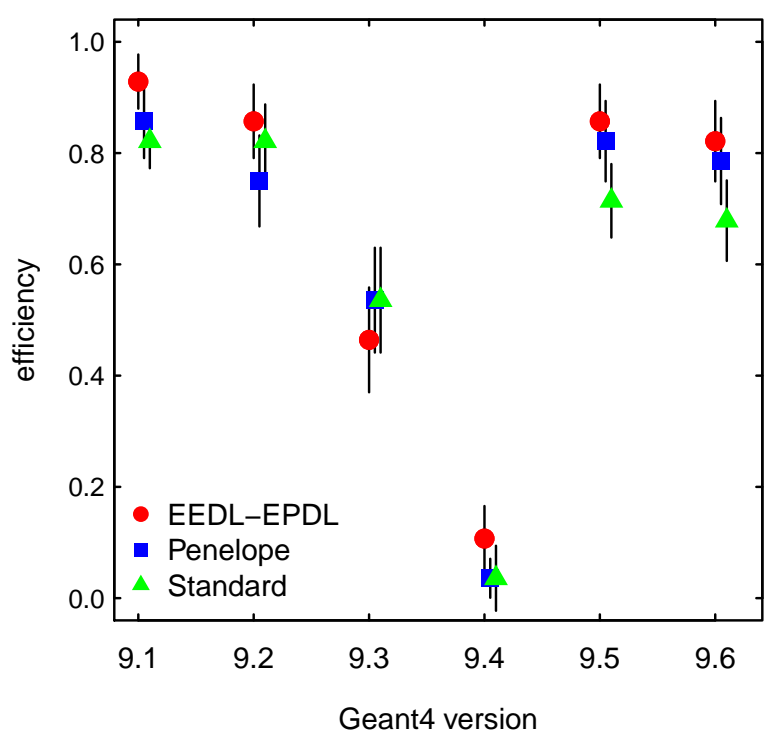

Fig. 4. Fraction of test cases in which Geant4-based simulations of the energy deposited by electrons in a bulk volume is found compatible with experimental data at 0.01 significance level, versus Geant 4 version. The experimental data are from [6]. Three Geant4 electromagnetic models (EEDL-EPDL, Penelope and Standard) were compared with experimental measurements.

A real-life example is extensively documented in [1] and is briefly discussed here. For this purpose one would consider the outcome of the same physics configuration in simulations involving different experimental scenarios: an example is shown in Fig. 1 and 4 , which correspond to the experimental setups sketched in Fig. 5and 6, respectively. The two setups are characterized by different sizes of the sensitive volumes where the deposited energy is collected: they correspond to a fine grained longitudinal segmentation in Fig. 1 1 and to a coarse granularity in Fig. 4. The compatibility of the simulation, encompassing identical physics settings, with experimental measurements is different in the two experimental configurations.

This issue should be properly taken into account, when dealing with the validation of observables produced by a simulation: the experimental context in which the observables have been produced, and the validity of the software is evaluated, should be specified.

\section{Elusive GoOdness}

It is common in the scholarly literature that the comparison of physics modeling calculations intended for Monte Carlo transport with experimental data is limited to visual appraisal only. Small size plots in logarithmic scale often hinder even a qualitative assessment. As an example of this practice, one could consider Fig. 6 of [8], where theoretical calculations of cross sections for the ionization of the $\mathrm{K}$ shell of various materials by electron impact are qualitatively compared to experimental measurements. The evaluation of the validity of the calculation is entirely left to a subjective visual appraisal of a plot, represented in logarithmic scale spanning several orders of magnitude. The original reference [8] does not report any

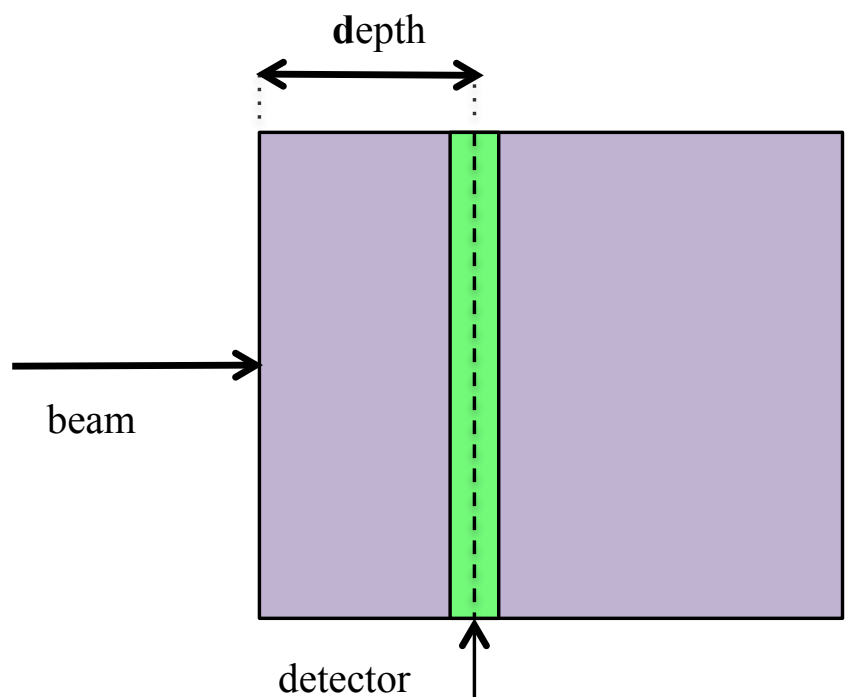

Fig. 5. The detector configuration corresponding to the experimental setup of [4] for the validation of the simulated longitudinal energy deposition. The length indicated as "depth" in the figure represents the longitudinal coordinate associated with the energy deposited in a thin detector. This configuration corresponds to the validation results illustrated in Fig. 1 .

quantitative estimate of the compatibility of the calculations described in the paper with experimental data, nor documents objectively whether these calculations achieve better compatibility with experimental data than other calculations available in the literature. In such a situation it is difficult for a Monte Carlo simulation user to discriminate whether the model of [8] satisfies the requirements of his or her experimental scenario, and whether it represents the state of the art or other physics models would ensure superior simulation accuracy.

The ionization of atomic shells by electron impact has recently been the subject of a thorough validation study, which will be documented in detail in a forthcoming publication

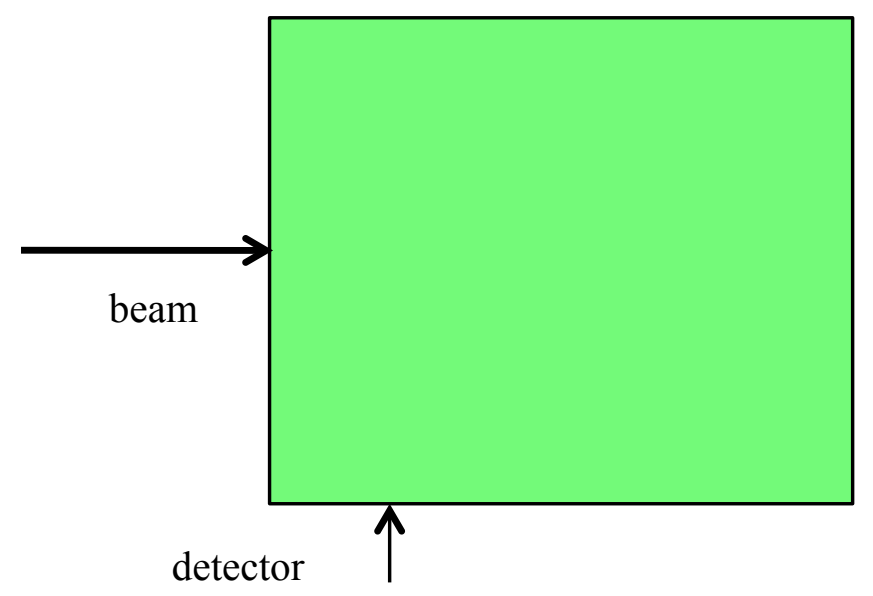

Fig. 6. The detector configuration corresponding to the experimental setup of [6] for the validation of the simulated energy deposited in a bulk volume. This configuration corresponds to the validation results illustrated in Fig. 4 


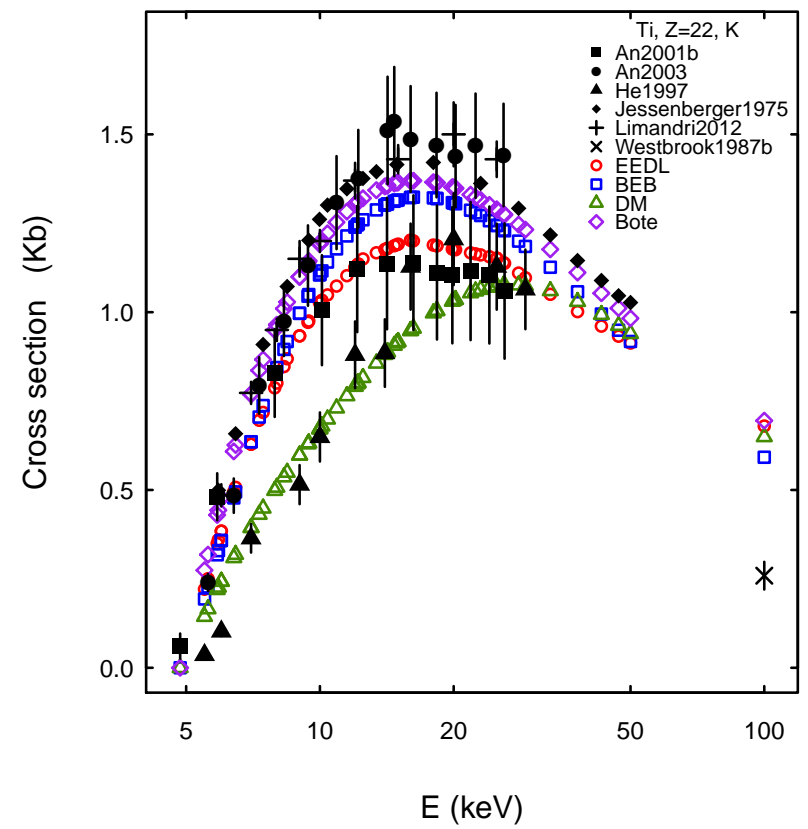

Fig. 7. Electron impact ionization cross section of titanium $\mathrm{K}$ shell as a function of energy: experimental data (filled symbols) and calculation models (open symbols).

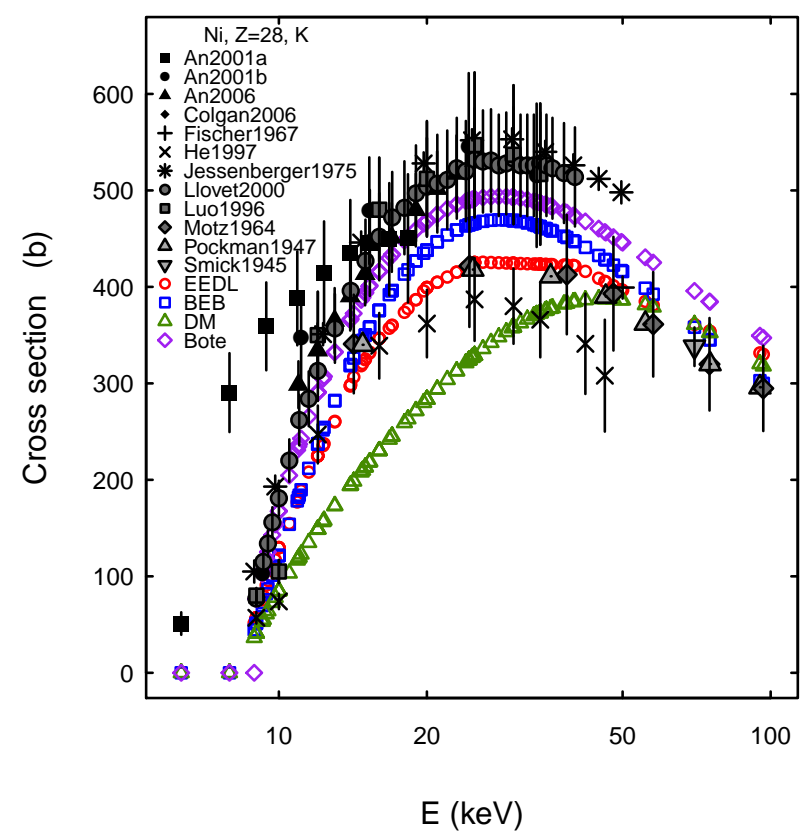

Fig. 8. Electron impact ionization cross section of nickel $\mathrm{K}$ shell as a function of energy: experimental data (filled symbols) and calculation models (open symbols).

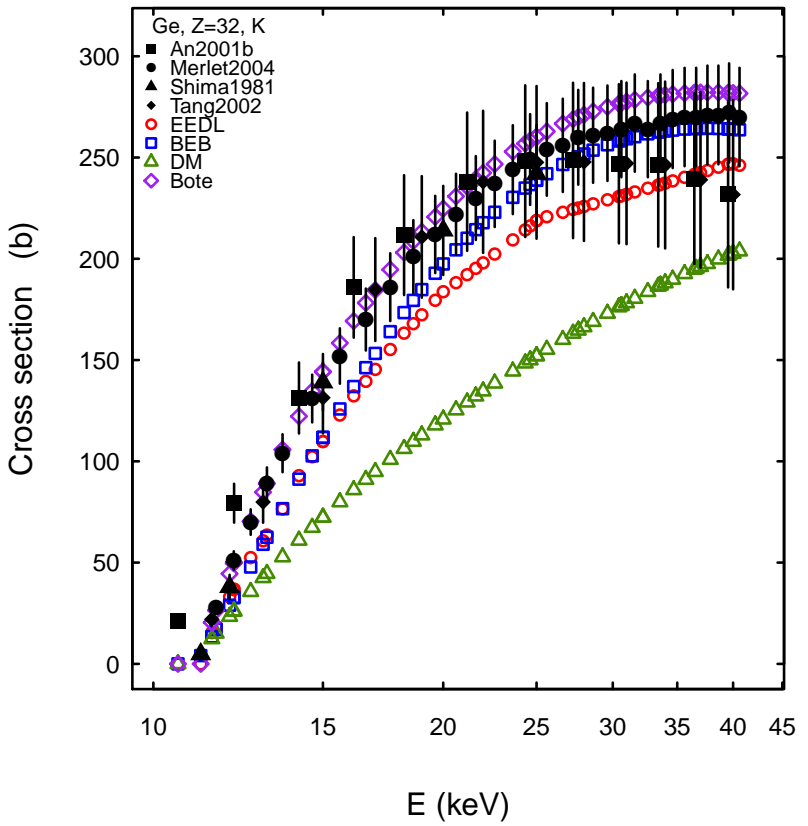

Fig. 9. Electron impact ionization cross section of germanium $\mathrm{K}$ shell as a function of energy: experimental data (filled symbols) and calculation models (open symbols).

involving the authors of the present paper. Some highlights are summarized here with the purpose of illustrating methods and achievements of a quantitative process for the validation of physics models implemented in Monte Carlo simulation systems and the comparison of different modeling alternatives.

Figs. 7 to 9 show the cross section calculations of $[8]$ along with other cross section calculations and experimental data. The alternative cross section calculations are based on EEDL (Evaluated Electron Data Library) [9], on the BinaryEncounter-Bethe model [10] and the Deutsch-Märk model [11]. The complete bibliographical information of the experimental data will be documented in the above mentioned forthcoming journal publication.

A two-stage statistical analysis over a large experimental data sample assesses objectively the physics capabilities and relative merits of the various models: it encompasses first a goodness-of-fit tests (here a $\chi^{2}$ test) to determine the compatibility of each calculated cross section with experimental data, then a categorical analysis based on contingency tables to identify whether a given model exhibits a significantly better compatibility with experiment than alternative models. This process identifies the calculation model of $[8]$ as significantly more accurate than the other alternatives at reproducing experimental K shell cross sections for electron impact ionization. The main outcome of the statistical analysis is summarized in Table If a variety of tests, all resulting in p-values smaller than the significance level of the test set at 0.01 , assess that the model of [8] results in significantly different compatibility with experiment with respect to cross sections based on EEDL.

\section{CONClusion}

A collection of scenarios discussed in this paper highlight the role of statistical methods to quantify the compatibility of 
TABLE I

P-VALUES RESULTING FROM CONTINGENCY TABLES COMPARING THE COMPATIBILITY WITH EXPERIMENT OF K SHELL CROSS SECTIONS FOR ELECTRON IMPACT IONIZATION BASED ON [8] AND EEDL

\begin{tabular}{lc}
\hline Test & p-value \\
\hline Fisher & 0.0098 \\
$\chi^{2}$ & 0.0076 \\
Barnard & 0.0079 \\
\hline
\end{tabular}

simulation with experimental data.

Quantitative comparisons with experiment are critical to appraise the reliability of the physics models implemented in particle transport codes and to determine the relative merits of alternative modeling options. Statistical methods are also helpful to monitor the functional quality of the code in the course of its evolution.

The validation of observables produced by Monte Carlo simulation systems is related both to the physics modeling embedded in the simulation and to the experimental configuration where the observable is produced. An identical physics configuration may result in largely different compatibility with experiment in different experimental scenarios.

Software verification and validation, in particular of physics models in simulation systems, can be facilitated by sound software design and other established best practices in software development.

\section{ACKNOWLEDGMENT}

The CERN Library has provided helpful assistance and essential reference material for this study. S. H. Kim thanks the INFN Section of Genova and CERN PH/SFT group for hosting his research activity in summer 2013.

\section{REFERENCES}

[1] M. Batic, G. Hoff, M. G. Pia, P. Saracco, and G. Weidenspointner, "Validation of Geant 4 simulation of electron energy deposition" IEEE Trans. Nucl. Sci., vol. 60, no. 4, pp. 2934-2957, 2013.

[2] S. Agostinelli et al., "Geant4 - a simulation toolkit" Nucl. Instrum. Meth. A, vol. 506, no. 3, pp. 250-303, 2003.

[3] J. Allison et al., "Geant4 Developments and Applications" IEEE Trans. Nucl. Sci., vol. 53, no. 1, pp. 270-278, 2006.

[4] G. Lockwood et al., "Calorimetric Measurement of Electron Energy Deposition in Extended Media - Theory vs Experiment", SAND790414 Report, Sandia National Laboratories, Albuquerque, 1980.

[5] M. Fowler, "Refactoring: improving the design of existing code", Addison-Wesley, 1999.

[6] G. J. Lockwood et al., "Electron Energy and Charge Albedos Calorimetric Measurement vs Monte Carlo Theory, SAND80-1968 Report, Sandia National Laboratories, Albuquerque, 1981.

[7] IEEE Computer Society, "IEEE Standard for Software Verification and Validation", IEEE Std 1012-2004, Jun. 2005.

[8] D. Bote and F. Salvat, "Calculations of inner-shell ionization by electron impact with the distorted-wave and plane wave Born approximation", Phys. Rev. A, vol. 77, p. 042701, 2008.

[9] S. T. Perkins et al., "Tables and Graphs of Electron-Interaction Cross Sections from $10 \mathrm{eV}$ to $100 \mathrm{GeV}$ Derived from the LLNL Evaluated Electron Data Library (EEDL)", UCRL-50400 Vol. 31, 1997.

[10] Y. K. Kim and M. E. Rudd, "Binary-encounter-dipole model for electron-impact ionization by electron impact," Phys. Rev. A, vol. 50, pp. 3954-3967, 1994.

[11] H. Deutsch, P. Scheier, K. Becker, T. D. Märk, "Revised high energy behavior of the Deutsch-Märk (DM) formula for the calculation of electron impact ionization cross sections of atoms," Int. J. Mass Spectrom., vol. 233, pp. 13-17, 2004. 